\title{
Long-term changes in bone metabolism, bone mineral density, quantitative ultrasound parameters, and fracture incidence after spinal cord injury: a cross-sectional observational study in 100 paraplegic men
}

\author{
Yvonne Zehnder · Markus Lüthi · Dieter Michel \\ Hans Knecht · Romain Perrelet · Isolde Neto \\ Marius Kraenzlin · Guido Zäch · Kurt Lippuner
}

Received: 7 February 2003 / Accepted: 15 September 2003/Published online: 13 January 2004

(C) International Osteoporosis Foundation and National Osteoporosis Foundation 2004

\begin{abstract}
To study the time course of demineralization and fracture incidence after spinal cord injury (SCI), 100 paraplegic men with complete motor loss were investigated in a cross-sectional study 3 months to 30 years after their traumatic SCI. Fracture history was assessed and verified using patients' files and X-rays. BMD of the lumbar spine (LS), femoral neck (FN), distal forearm (ultradistal part $=$ UDR, $1 / 3$ distal part $=1 / 3 R$ ), distal tibial diaphysis (TDIA), and distal tibial epiphysis (TEPI) was measured using DXA. Stiffness of the calcaneus (QUI.CALC), speed of sound of the tibia (SOS.TIB), and amplitude-dependent SOS across the proximal phalanges (adSOS.PHAL) were measured using QUS. Z-Scores of BMD and quantitative ultrasound (QUS) were plotted against time-since-injury and compared among four groups of paraplegics stratified according to time-since-injury ( $<1$ year, stratum I; 1-9 years, stratum II; 10-19 years, stratum III; 2029 years, stratum IV). Biochemical markers of bone turnover (deoxypyridinoline/creatinine (D-pyr/Cr), osteocalcin, alkaline phosphatase) and the main parameters of calcium phosphate metabolism were measured. Fifteen out of 98 paraplegics had sustained a total of 39 fragility fractures within 1,010 years of
\end{abstract}

Both authors, Y. Zehnder and M. Lüthi, have equally contributed to this work.

Y. Zehnder · D. Michel · H. Knecht · G. Zäch

Swiss Paraplegic Center, CH-6207, Nottwil, Switzerland

M. Lüthi $\cdot$ R. Perrelet · I. Neto $\cdot$ K. Lippuner $(\bowtie)$

Osteoporosis Unit, University Hospital of Berne,

CH-3010, Berne, Switzerland

E-mail: kurt.lippuner@insel.ch

Tel.: +41-31-6323185

Fax: + 41-31-6329596

M. Kraenzlin

Endocrine Unit and Department of Medicine,

University Hospital of Basel, CH-4031, Basel, Switzerland observation. All recorded fractures were fractures of the lower limbs, mean time to first fracture being $8.9 \pm 1.4$ years. Fracture incidence increased with time-after-SCI, from $1 \%$ in the first 12 months to $4.6 \%$ /year in paraplegics since $>20$ years $(p<.01)$. The overall fracture incidence was $2.2 \%$ /year. Compared with nonfractured paraplegics, those with a fracture history had been injured for a longer time $(p<.01)$. Furthermore, they had lower $Z$-scores at FN, TEPI, and TDIA $(p<.01$ to $<.0001)$, the largest difference being observed at TDIA, compared with the nonfractured. At the lower limbs, BMD decreased with time at all sites $(r=.49$ to .78 , all $p<.0001)$. At FN and TEPI, bone loss followed a $\log$ curve which leveled off between 1 to 3 years after injury. In contrast, $Z$-scores of TDIA continuously decreased even beyond 10 years after injury. LS BMD $Z$-score increased with time-since-SCI $(p<.05)$. Similarly to DXA, QUS allowed differentiation of early and rapid trabecular bone loss (QUI.CALC) vs slow and continuous cortical bone loss (SOS.TIB). Biochemical markers reflected a disproportion between highly elevated bone resorption and almost normal bone formation early after injury. Turnover declined following a log curve with time-after-SCI, however, D-pyr/Cr remained elevated in $30 \%$ of paraplegics injured $>10$ years. In paraplegic men early (trabecular) and persistent (cortical) bone loss occurs at the lower limbs and leads to an increasing fracture incidence with time-after-SCI.

Keywords Biochemical bone markers - Bone loss · DXA · Fracture incidence · Quantitative ultrasound $\cdot$ Spinal cord injury

\section{Introduction}

Bone loss after spinal cord injury (SCI) leads to a high incidence of fractures of the lower limbs in paraplegics 
$[1,2]$. Today, 60 years after Albright's first description of disuse osteoporosis [3], many questions regarding the cause, pattern, and time course of demineralization after SCI are still unresolved.

A few longitudinal studies have addressed the issue of bone turnover [4] or bone loss [5, 6, 7, 8] after SCI. Taken together, they suggest an extensive early demineralization after medullary lesion. These studies have usually been conducted on a small numbers of patients, and/or their duration was limited to the first few months after SCI, and/or they included an heterogeneous patient population (men and women, paraplegics and tetraplegics, complete and incomplete $\mathrm{SCI}$ ), and/or they did not adjust BMD for age and time-since-injury $[9,10,11,12$, $13,14]$.

The duration of bone loss after SCI and the infra- and supralesional, trabecular as well as cortical, bone loss patterns remain unknown in paraplegic men. Although the most common fractures after SCI are fractures of femur and tibia [15], no tibial BMD data have been available so far in these patients. In addition the question of whether alterations in qualitative bone properties (QUS data) are confined to the paralyzed limbs or affect the whole skeleton remains open, and data in patients after SCI are limited to the calcaneus [16].

Recent technical developments give a more detailed insight into the pathogenesis of osteoporosis in SCI patients. Regional high-resolution DXA allows infraand supralesional measurements of trabecular and cortical sites. Bone mineral density measurements at distal tibia, a standardized method established earlier by our group [17] to assess bone loss at a predominantly cortical site - the tibial diaphysis - and in an area containing a substantial amount of trabecular bone - the distal tibial epiphysis - in one single, normally weight-bearing, bone, are of particular interest in SCI patients. Quantitative ultrasound (QUS) may provide additional information with respect to qualitative bone properties such as elasticity and microstructure [18].

The aim of this cross-sectional study was to document the fracture history, the regional evolution of BMD (infra- and supralesional / trabecular and cortical) and QUS parameters as well as biochemical parameters of bone turnover in a large and homogeneous group of SCI patients.

\section{Patients and methods}

One hundred paraplegic men attending the outpatient clinic of the Swiss Paraplegic Center (SPC), Nottwil, Switzerland, were investigated between November 1997 and June 1999.

Inclusion criteria were male gender; complete motor posttraumatic medullary lesion between T1-L3 with total motor and sensory loss (corresponding to stage A according to the Frankel classification), or with total motor and partial sensory loss (Frankel stage B); age between 18 and 60 years; traumatic event leading to paraplegia after the age of 17 .

Exclusion criteria were use of drugs affecting bone metabolism (fluorides, bisphosphonates, vitamin D, calcitriol, anabolic steroids, chronic glucocorticoid use for more than 3 months, thyroid hor- mones, cytostatics, antiandrogens, lithium, chronic use of antiepileptics), known diseases affecting bone (algodystrophy, spondylitis ankylosans, rheumatoid arthritis, Paget's disease, osteogenesis imperfecta, hyperthyroidism, hyperparathyroidism, hypogonadism, hypercortisolism, malignant tumors, renal bone disease, chronic liver disease and post-transplantation bone disease).

All clinical investigations were conducted in accordance with the Declaration of Helsinki, and the present study protocol was approved by the Ethics Committee of the Canton of Lucerne (Switzerland). All patients gave their written informed consent before entering the study.

\section{Fracture history}

Subjects were asked about their fracture history and the exact mechanism leading to fracture. Traumatic fractures were distinguished from low-energy trauma events (i.e., osteoporotic fracture). Data were confirmed based on their medical records and X-rays.

\section{Dual-energy X-ray absorptiometry (DXA)}

The bone mineral density (BMD) of the lumbar spine (LS, second to fourth lumbar vertebrae), femoral neck (FN), distal forearm (ultradistal part $=$ UDR, $1 / 3$ distal part $=1 / 3 \mathrm{R}$ ), distal tibial diaphysis (TDIA) and distal tibial epiphysis (TEPI) [17] was measured using DXA (Hologic QDR 4500A; Hologic, Bedford, MA). BMD was expressed as $\mathrm{g} / \mathrm{cm}^{2}$ of hydroxyapatite and as $Z$-score (SD from the mean of a healthy male population of the same age). Four hundred normal Caucasian men, 20-80 years of age, living in the area of Berne, served as reference population. Peak bone mass (mean value \pm SD) was $1.100 \pm 0.139 \mathrm{~g} / \mathrm{cm}^{2}$ at $\mathrm{LS}$, $0.937 \pm 0.121 \mathrm{~g} / \mathrm{cm}^{2}$ at FN, $\quad 0.548 \pm 0.060 \mathrm{~g} / \mathrm{cm}^{2}$ at UDR, $0.817 \pm 0.053 \mathrm{~g} / \mathrm{cm}^{2}$ at $1 / 3 \mathrm{R}, \quad 0.927 \pm 0.119 \mathrm{~g} / \mathrm{cm}^{2}$ at TEPI, $1.375 \pm 0.118 \mathrm{~g} / \mathrm{cm}^{2}$ at TDIA. All scans were performed according to the manufacturer's guidelines. Peripheral bone measurements were carried out at the nondominant limb. Quality control was performed daily (anthropometric spine phantom supplied by the manufacturer): overall precision error was $0.3 \%$ in vitro and mean precision error in our hands is $1.1 \%$ in vivo (LS).

Quantitative ultrasound (QUS) of calcaneus, tibia, and phalanges

$Z$-scores of all QUS measurements were derived from the manufacturer's reference database.

\section{Calcaneus}

The quantitative ultrasound index (QUI.CALC; arbitrary units (AU)) was measured using the Sahara device (Hologic, Bedford, MA). QUI.CALC integrates the parameters, speed of sound (SOS.CALC; $\mathrm{m} / \mathrm{s}$ ) and broadband ultrasound attenuation (BUA.CALC; $\mathrm{dB} / \mathrm{MHz}$ ) across the calcaneus according to the formula $\mathrm{QUI}=(0.41 \times \mathrm{BUA})+(0.41 \times \mathrm{xOS})-571$. Typically, QUI ranges from 80 to $120 \mathrm{AU}$ in healthy young men. In our hands, precision error is $1.8 \pm 0.8 \%$ (6 heels measured 11 times over 14 days).

\section{Tibia}

Speed of sound (SOS.TIB; m/s) was measured at the tibial crest using a Sound Scan 2000 (Myriad Ultrasound Systems, Rehovot, Israel), according to the manufacturer's specifications. The average of the five highest readings was considered as the measured value. Short-term in vivo precision error in our hands is around $2 \%$. Reported standardized coefficient of variation for this method is $1.6 \%[19]$. 


\section{Phalanges}

Amplitude-dependent speed of sound SOS (adSOS.PHAL; m/s) was measured across the 2 nd to 5 th proximal phalanges of the nondominant hand using the Sonic DBM 1200 Device (IGEA, Italy). Short-term in vivo precision error in our hands was around $1 \%$-i.e., similar to that reported by others [20].

Biochemical parameters

Blood

Venous blood was drawn in the morning after an overnight fast. In all patients, laboratory analyses were performed either within the $2 \mathrm{~h}$ after drawing of blood or on thawed plasma that had been stored at $-80^{\circ} \mathrm{C}$ prior to analyses. Serum concentrations of total calcium $(\mathrm{Ca}$; normal range (NR) $2.15-2.55 \mathrm{mmol} / \mathrm{l}$ ), phosphorus (P; NR 0.87-1.45 mmol/l), creatinine (Cr; NR 44-80 $\mu \mathrm{mol} / \mathrm{l})$, and plasma total alkaline phosphatase (ALP; NR 39-117 U/1) were measured using standard laboratory techniques and normal ranges from the biochemistry laboratory of the University Hospital of Berne. Ionized calcium $(\mathrm{Ca}++$; NR $1.15-1.30 \mathrm{mmol} / \mathrm{l})$ was measured in whole blood using an ion-selective electrode (Ciba Corning Diagnostics, Medford, MA). The serum concentrations of intact PTH (PTH; Allegro, Nichols Institute Diagnostics, San Juan Capistrano, CA; NR 10-65 pg/ml), 25-hydroxyvitamin D (25OHD; Nichols Institute Diagnostics; NR 6-40 ng/ml), osteocalcin (OC; Nichols Institute Diagnostics; NR 11.1-32.2 ng/ml), and serum-free testosterone ( $\mathrm{T}$; Diagnostic Systems Laboratories, TX; NR $8.7-54.7 \mathrm{pg} / \mathrm{ml}$ ) were measured by radio-immunoassays and manufacturers' normal ranges.

Urine

Two-hour fasting urine samples (second morning voided urine) were analyzed for calcium (atomic absorptiometry) and creatinine (standard colorimetric assay based upon the Jaffe method). Urinary deoxypyridinoline (D-pyr) was measured by reverse-phase ionpaired HPLC (Crosslinks, BioRad, Germany). Results were expressed as ratios of $\mathrm{Ca} / \mathrm{Cr}(\mathrm{NR} 0.1-0.32 \mathrm{mmol} / \mathrm{mmol})$ and D-pyr/ $\mathrm{Cr}$ (NR 9-20 nmol/mmol), based on manufacturer's normal range. Twenty-four-hour urinary excretion of calcium $\left(\mathrm{U}_{\mathrm{Ca}} \mathrm{xV}\right.$; NR 2.5$8.0 \mathrm{mmol} / 24 \mathrm{~h})$ and creatinine $\left(\mathrm{U}_{\mathrm{Cr}} \mathrm{xV}\right.$; NR $6,000-18,000 \mathrm{mmol} /$ $24 \mathrm{~h}$ ) was measured using standard laboratory techniques.

Nutrition and lifestyle questionnaire

Dietary calcium intake was determined using food frequency questionnaires and indicated as $\mathrm{mg}$ of calcium per day. Current alcohol consumption was expressed as standard drinks per day. Lifetime amount of smoking was presented in pack-years, smokers were defined as having smoked 5 pack-years or more in their lives. Assessment of physical activity included estimation of daily hours of sitting, current standing activities ( $\mathrm{min} /$ day), and total time spent with sportive activities (hr/week). Data on duration of initial bed rest after SCI was taken from medical records.

\section{Statistical analysis}

Statistics were performed using a statistical software package (Statview 4.1; Abacus Concept, Berkeley, CA). Values are given as mean \pm SEM. In accordance with a recently published study [11], patients were stratified into the following four groups according to time-since-injury: i.e., < 1 year (stratum I), 1 to 9 years (stratum II), 10 to 19 years (stratum III), and 20 to 29 years (stratum IV) postinjury. Linear or -where more appropriate-logarithmic regression was used to describe the relationship between biochemical markers, bone density, and quantitative ultrasound parameters, on the one hand, and time-since-SCI, on the other. Multiple regression analysis was used to define determinants of biochemical indices, BMD, and QUS.

Fracture history was documented based on total number of fractures and fracture episodes: multiple fractures occurring at a single traumatic event were counted as one fracture episode. Fractures due to a high-energy trauma and fractures that occurred at the time of SCI were not included in the analysis. The rate of anamnestic fractures (\%) was calculated for each stratum by timesince-injury.

\section{Results}

\section{Patient characteristics}

One hundred male patients with complete motor posttraumatic medullary lesion between T1-L3 and either total motor and sensory loss ( $n=94$, Frankel stage A) or total motor and partial sensory loss $(n=6$, Frankel B) were screened for participation to this cross-sectional study (Table 1 ). Of the patients, $21 \%$ had a lesion $\mathrm{T} 1-$ T5, 44\% T6-T10, and 35\% T11-L3.

Mean $( \pm$ SEM $)$ estimated calcium intake was $1,293 \pm 67.3 \mathrm{mg} /$ day (range $250-4,260 \mathrm{mg} /$ day). $\mathrm{Pa}-$ tients spent an average of $13.5 \pm 0.3 \mathrm{~h}$ per day sitting in the wheelchair (range $6.0-18.0)$. They did $(n=51)$ or did not $(n=49)$ regularly perform passive weight-bearing standing with the aid of a standing device: 22 men reported standing for less than 30 min per day, whereas 24 men reported doing so for 30-60 min and 5 for more than $1 \mathrm{~h}$. Sportive activities (e.g., basketball, hand-biking, swimming, tennis, weight lifting, skiing) were performed by 67 men; 42 did so with high intensity ( $>3 \mathrm{~h} /$ week), and 25 with moderate intensity ( $<3 \mathrm{~h} /$ week). Fourty-nine men were smokers. Alcohol consumption was low to moderate ( $\leq 2$ standard drinks / day) in 88 men, and more than 2 standard drinks / day in 12 men. Two patients were excluded based on the exclusion criteria (one hypogonadism, one hyperparathyroidism).

\section{Fracture history}

Fifteen of the 98 paraplegic men included had sustained 22 episodes with a total of 39 fractures within 1,010

Table 1 Characteristics of study population $(n=100)$. SCI spinal cord injury

\begin{tabular}{lccc}
\hline Parameter & Mean \pm SEM & Median & Patients' range \\
\hline Age (years) & $38.0 \pm 0.97$ & 37.3 & $18.3-59.9$ \\
Height $(\mathrm{cm})$ & $177.1 \pm 0.78$ & 177.0 & $160-196$ \\
Weight $(\mathrm{kg})$ & $71.4 \pm 1.07$ & 71.0 & $45-98$ \\
Body mass index $\left(\mathrm{kg} / \mathrm{m}^{2}\right)$ & $22.7 \pm 0.31$ & 22.7 & $15.4-30.1$ \\
Age at injury (years) & $27.5 \pm 0.93$ & 24.5 & $17.0-57.8$ \\
Duration of SCI (years) & $10.4 \pm 0.79$ & 9.5 & $0.1-29.5$ \\
Initial bed rest (months) & $4.3 \pm 0.43$ & 3.0 & $0.5-28.0$ \\
\hline
\end{tabular}


years of observation (mean $10.4 \pm 0.79$ years observation per patient). Both the total number of individual fractures as well as the number of fracture episodes increased regularly with the number of years since injury (Table 2; Fig. 1). Mean time to first fracture after SCI was $8.9 \pm 1.4$ years (median 8.5 years). Although most fractures were due to a fall from the wheelchair, no fracture of the upper limbs was registered: 9 femur (neck 3 , shaft 3, supracondylar 3), 3 knee (condylar 2, patellar 1), 13 tibia, 9 ankle (fibular 8, calcaneal 1), and 4 foot (metatarsal 3, toe 1) fractures occurred. The mean yearly fracture incidence increased significantly $(p<0.01)$ with time-after-SCI from 1\%/year (stratum I) to $4.6 \%$ year (stratum IV), with an overall mean fracture incidence of $2.2 \%$.

Paraplegic men with fractures $(n=15)$ had been injured for a significantly longer time $(15.7 \pm 1.9$ vs $9.3 \pm 0.8$ years, $p<0.01)$ than men without fractures. There was no significant difference between the two groups with regard to age, calcium intake, physical activity, smoking, or alcohol consumption habits.

Bone mineral density

Bone mineral density measurements were performed in 90/98 patients at the lumbar spine (LS), in 91/98 at the radius (ultradistal radius UDR and shaft 1/3R), in 94/98 at the femoral neck (FN), in 93 and $92 / 98$ at the tibial diaphysis (TDIA) and tibial epiphysis (TEPI), respectively. Not evaluable scans were due to metallic implants (LS), positioning problems (UDR, 1/3R), periarticular ossification ( $\mathrm{FN}$ ), and positioning problems due to the spasticity of the legs (TDIA, TEPI).

Individual BMD $Z$-scores as a function of time-afterSCI are depicted in Fig. 2. The comparison of mean $\mathrm{BMD}, Z$-scores, and the number and percentage of

Table 2 Fracture incidence, BMD, and QUS, stratified by time-since-injury in 98 paraplegics (after exclusion of patients nos. 41 and 46 due to secondary osteoporosis). $p$ Values are post hoc Fisher PLSD

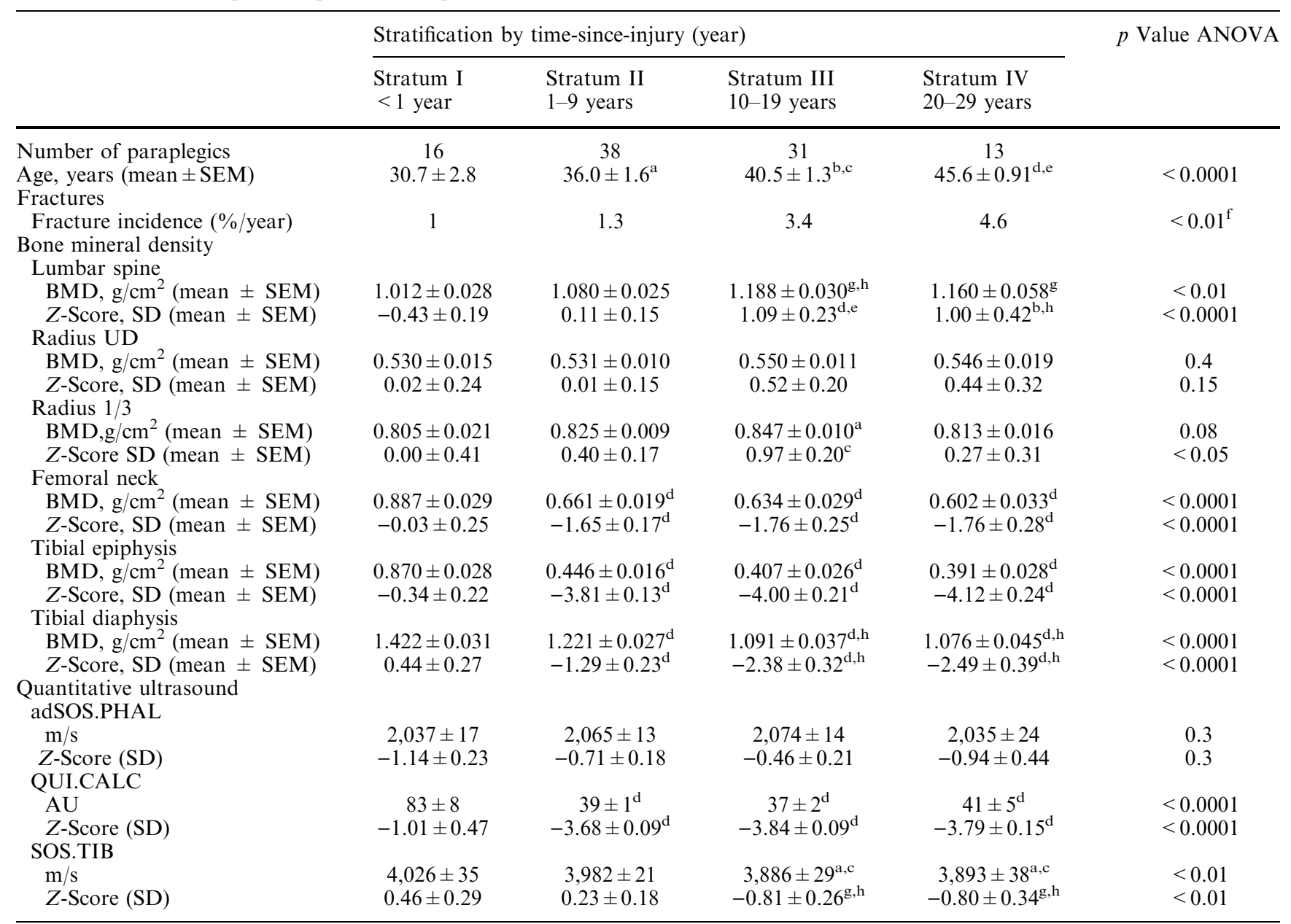

\footnotetext{
${ }^{\mathrm{a}} p<0.05$ vs $<1$ year;

${ }^{\mathrm{b}} p<0.001$ vs $<1$ year;

c $p<0.05$ vs $1-9$ years;

${ }_{p}<0.0001$ vs $<1$ year;
}
e $p<0.001$ vs $1-9$ years;
by $\chi$-square test;
${ }_{p}<0.01$ vs $<1$ year;
${ }^{\mathrm{h}} p<0.01$ vs $1-9$ years




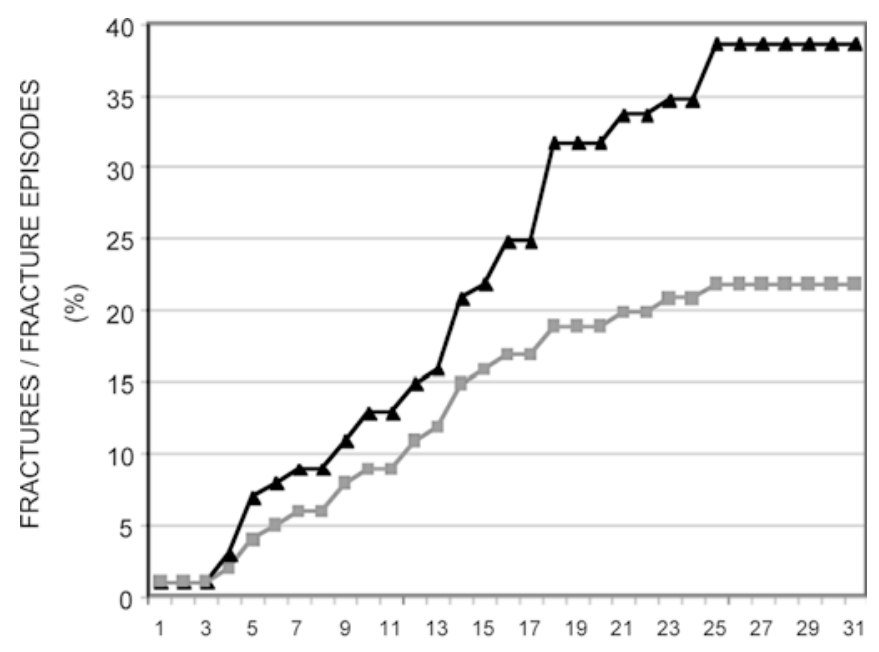

TIME SINCE SPINAL CORD INJURY (YEARS)

Fig. 1 Cumulative fracture rate (triangles) and fracture incidence (rectangles) observed in 1,010 patient-years among 98 paraplegic men $0-30$ years after spinal cord injury

osteoporotic patients within the various strata postinjury is shown in Table 2.

At LS, a significant positive correlation between $Z$-scores and time-after-injury was observed $(r=0.43$; $p<0.0001)$. The mean lumbar $Z$-score was slightly negative in recently injured men (stratum I), identical to age-matched normal controls between 1 and 9 years postinjury (stratum II), and +1 SD above age-matched normal controls after 10 years or more of injury.

At the radius, the $Z$-scores at both sites were comparable to age-matched normal controls in strata I and II, and +0.5 to $+1 \mathrm{SD}$ above age-matched normal controls after 10 years of injury.

At the lower limbs, BMD decreased dramatically with time at all sites, correlation coefficients ranging from 0.49 to 0.78 (all $p<0.0001$ ). At FN and TEPI, bone loss followed a log curve which leveled off between 1 and 3 years after injury. In contrast, $Z$-scores of TDIA decreased progressively even beyond 10 years after injury.

These results were tested for several possible determinants of bone loss other than time-since-SCI: there were no significant correlations of BMD $Z$-scores at any site with current calcium intake, alcohol consumption, current standing, sportive activity, level of spinal cord lesion (T1-T10 = high; T11-L3 = low) and presence or absence of spasticity. Only smokers $(n=47)$ had significantly lower BMD $Z$-scores compared with nonsmokers $(n=51)$ at the infralesional sites FN $(-1.7 \pm 0.18 \mathrm{SD}$ vs $-1.1 \pm 0.18 \mathrm{SD} ; p<0.05)$, TEPI $(-3.8 \pm 0.23 \mathrm{SD}$ vs $2.9 \pm 0.23 \mathrm{SD} ; p<0.01)$ and TDIA $(-1.8 \pm 0.26 \mathrm{SD}$ vs $1.1 \pm 0.23 \mathrm{SD} ; p<0.05)$ but not at the supralesional LS and forearm sites. Patients with anamnestic fractures after SCI had significantly lower $Z$-scores at the infralesional sites FN $(-2.3 \pm 0.36 \mathrm{SD}$ vs $-1.3 \pm 0.14 \mathrm{SD} ; p<0.01)$, TEPI $(-4.6 \pm 0.34 \mathrm{SD}$ vs $-3.1 \pm 0.18 \mathrm{SD} ; p<0.001)$ and TDIA $(-3.1 \pm 0.40 \mathrm{SD}$ vs $-1.2 \pm 0.18 \mathrm{SD} ; p<0.0001)$ but not at the supralesional LS and forearm sites.
Quantitative ultrasound

Analogously to BMD at tibial epiphysis, $Z$-scores of QUI.CALC decreased following a logarithmic curve with time-since-injury which leveled off 6 to 12 months postinjury, whereas $Z$-scores of SOS.TIB decreased more linearly with time-after-injury, similarly to BMD of the tibial diaphysis. Z-scores of ad.SOS.PHAL did not significantly change with time-after-injury over the time period (Table 2; Fig. 3).

These results were tested for several possible determinants of bone loss other than time-since-SCI: there were no significant correlations of QUS Z-scores at any site with current calcium intake, alcohol consumption, current standing, sportive activity, level of spinal cord lesion, presence or absence of spasticity, smoking, or history of fracture(s) after SCI.

QUI.CALC was positively correlated with BMD of FN, TDIA, and TEPI ( $p<0.0001$ for all regressions). The highest coefficient of correlation was achieved with BMD at TEPI $(r=0.72)$. Similarly, SOS.TIB was positively correlated with BMD at infralesional sites $(p<0.005)$, the highest correlation being found with BMD at TDIA $(r=0.53)$. AdSOS.PHAL correlated positively with BMD of both subregions of the arms: i.e., $1 / 3 \mathrm{R}$ and UDR $(r=0.24 ; p<0.05$, for both).

\section{Biochemistry}

Generally speaking, markers of bone resorption (Dpyr $/ \mathrm{Cr}, \mathrm{Ca} / \mathrm{Cr}$ ) were dramatically increased in recently injured paraplegics, whereas bone formation markers (ALP, OC) were either slightly elevated or within the upper reference range (Fig. 4). While the majority of patients injured for more than 1 year showed normal bone formation markers, bone resorption estimated by $\mathrm{D}-\mathrm{pyr} / \mathrm{Cr}$ ratio remained elevated in $50 \%$ and $30 \%$ of paraplegics in stratum II (1-9 years post-SCI) and strata III-IV (10 years or more post-SCI), respectively.

In recently injured paraplegics, serum $\mathrm{Ca}^{++}, \mathrm{P}$, and $\mathrm{U}_{\mathrm{Ca}} \mathrm{xV}$ were elevated and serum levels of intact PTH initially suppressed. In patients $>1$ year after SCI, these parameters were within normal range (data not shown).

Serum $\mathrm{Ca}^{++}$and OC decreased significantly with age ( $r=0.34$ and 0.44 , respectively; both $p<0.001)$ and with time-after-injury $(r=0.34$ and 0.37 , respectively; both $p<0.001)$. Serum intact PTH increased with age $(r=0.42 ; p<0.0001)$ and with time-since-injury $(r=0.44$; $p<0.0001$ ). D-pyr/Cr, Ca/Cr, ALP, $\mathrm{P}$, and $\mathrm{U}_{\mathrm{Ca}} \mathrm{xV}$ decreased with time-since-injury but not with age. Serum 25OHD and $\mathrm{U}_{\mathrm{Cr}} \mathrm{xV}$ were independent of both timesince-injury and age. In three cases, 25OHD levels were slightly below the reference range. No other parameters such as weight, nutrition, or physical activity were found to influence biochemical markers of bone metabolism.

After controlling for age and time-since-injury, patients with anamnestic fracture(s) after SCI had signifi- 
Fig. 2 BMD $Z$-scores (SD) at various skeletal sites as a function of time since spinal cord injury in 98 paraplegic men
LUMBAR SPINE

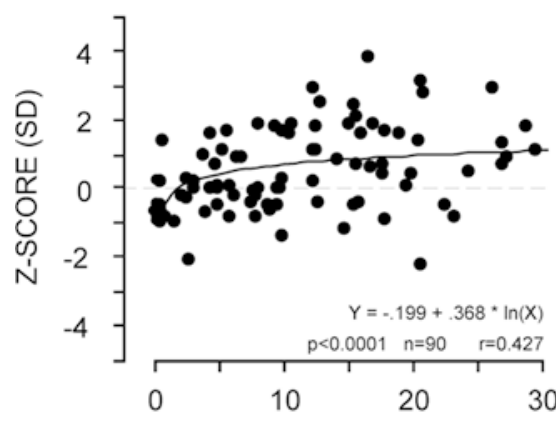

RADIUS $1 / 3$

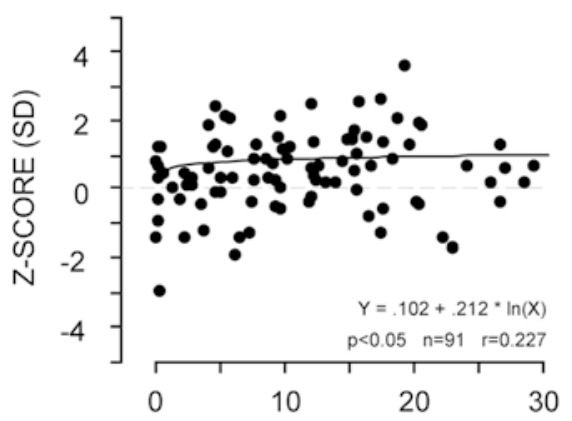

RADIUS UD

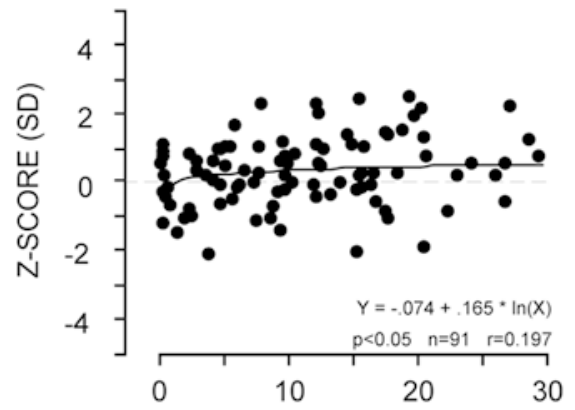

YEARS SINCE SPINAL CORD INJURY
FEMORAL NECK

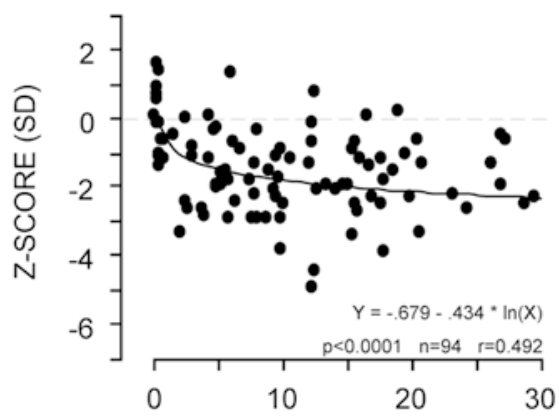

TIBIAL DIAPHYSIS

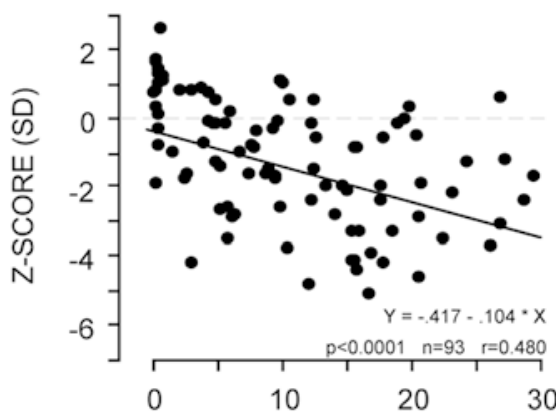

TIBIAL EPIPHYSIS

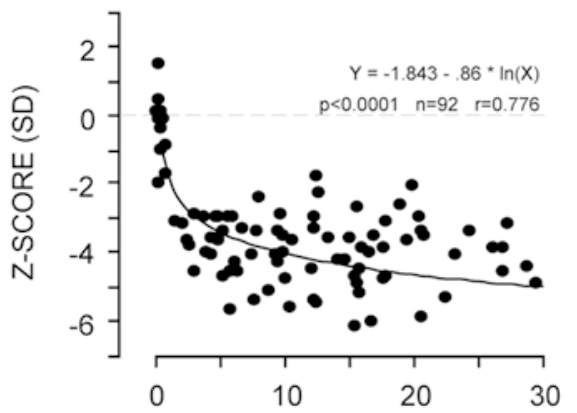

YEARS SINCE SPINAL CORD INJURY cantly lower OC serum concentrations $(p<0.05)$ and significantly higher D-pyr/Cr $(p<0.05)$.

\section{Discussion}

This cross-sectional study is the first to document the fracture history, regional evolution of BMD (infra- and supralesional / trabecular and cortical) and QUS parameters as well as biochemical parameters of bone turnover in a large (98 patients) and homogeneous (men with complete motor post-traumatic medullary lesion) group of SCI patients.

In the present study, fractures occurred exclusively at the lower extremities, numerically more frequently at the tibia, an observation which is in accordance with that of others [2]. The fractures did not occur in the first 3 years after injury, although $40 \%$ of trabecular bone had already been lost at that time and the curve of trabecular bone loss had even leveled off. The median duration to first fragility fracture was 8.5 years, a point in time where the BMD of the tibial cortex had reached a mean $Z$-score of $-2.5 \mathrm{SD}$. In addition, the largest difference in terms of BMD $Z$-score between paraplegics with and those without a history of fractures, was found at the tibial diaphysis (i.e., 1.9 SD), a predominantly cortical site. The chronic cortical demineralization process, including at the tibia, could at least partly explain the late but frequent occurrence of fragility fractures in the present population of paraplegic men. These fracture 
Fig. 3 Quantitative ultrasound parameters (absolute values and corresponding $Z$-scores) at various skeletal sites as a function of time since spinal cord injury in 98 paraplegic men

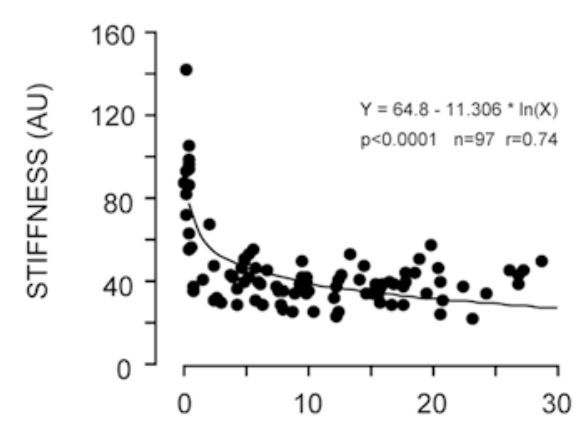

CALCANEUS

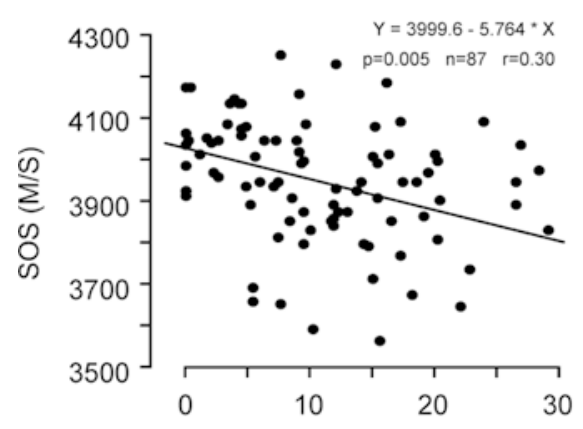

PHALANGES

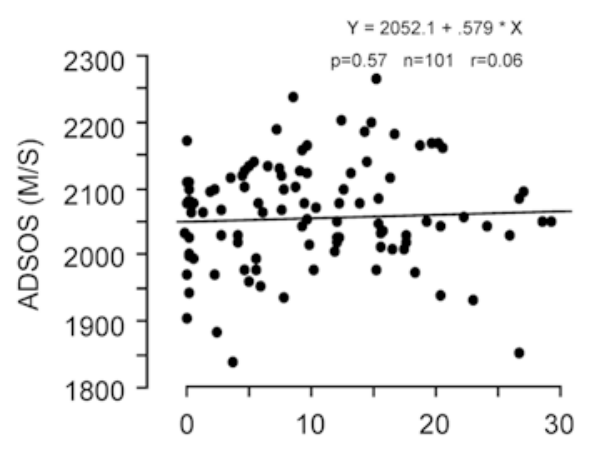

YEARS SINCE SPINAL CORD INJURY
TIBIA
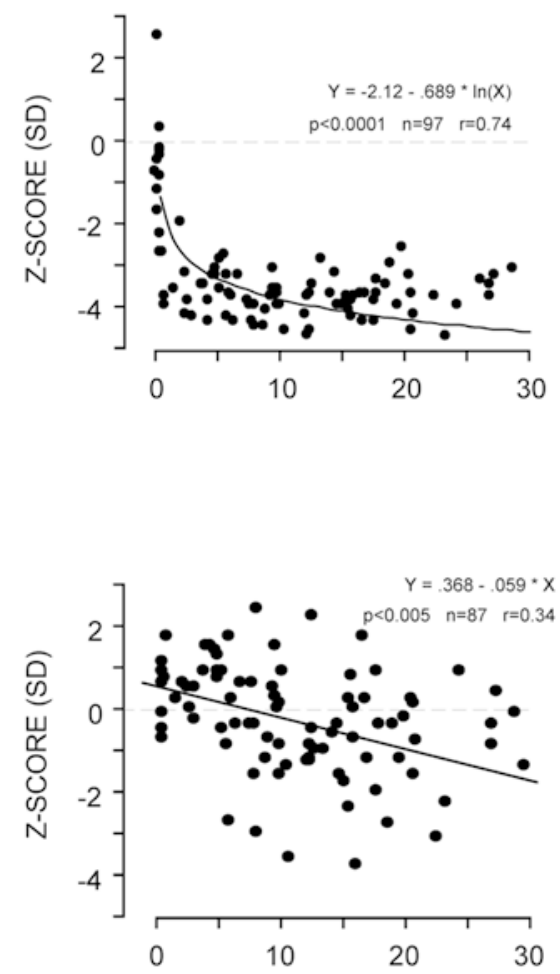

0

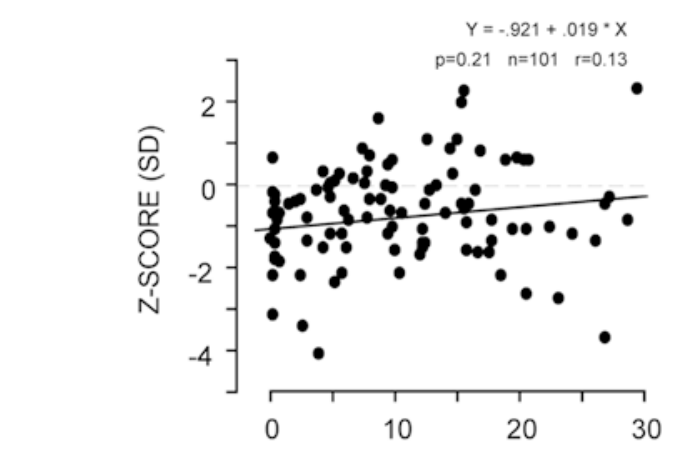

YEARS SINCE SPINAL CORD INJURY data compare well with those recently collected from 438 paraplegics of the Danish Paraplegic Association and from 654 randomly selected normal controls using selfadministered questionnaires[2]. They found a crude fracture incidence of $2 \%$ per year in paraplegics: i.e., a twofold increase compared with controls $(p<0.001)$ which is similar to the yearly incidence of fracture episodes of $2.2 \%$ reported in this study. They reported a median time-since-injury of 11 years in females and 12 years in males, which is slightly longer than reported here and may be due to the neurologic heterogeneity of the included patients (complete or partial paralysis). Earlier studies reported fracture incidences between $4 \%$ $[21,22]$ and $11 \%$ [23], without mentioning the time point of observation after SCI.
A new finding is the differential pattern of sublesional bone loss in paraplegics with respect to trabecular and cortical bone compartments. Until today, BMD in paraplegics has not been measured in skeletal sites more distal to the femur. In this study we measured the BMD at distal tibia [17]. The BMD at the mainly trabecular TEPI site and at the mainly cortical TDIA site, with all the precautions required due to the cross-sectional design, seems to have followed a different decrease pattern in paraplegic men: the tibial epiphysis, and to a lesser extent femoral neck - both containing a substantial part of trabecular bone - showed early and rapid demineralization which leveled off within the first 3 years after injury. In contrast, the tibial diaphysis - containing almost exclusively cortical bone [17] — showed a slower but 
Fig. 4 Evolution of biochemical parameters of bone resorption and bone formation as a function of time since spinal cord injury in 98 paraplegic men
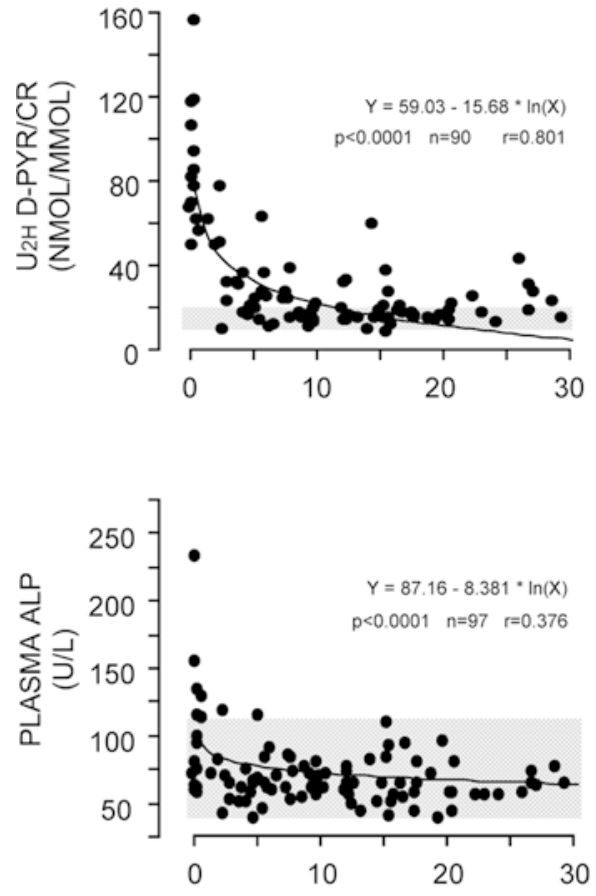

YEARS SINCE SPINAL CORD INJURY
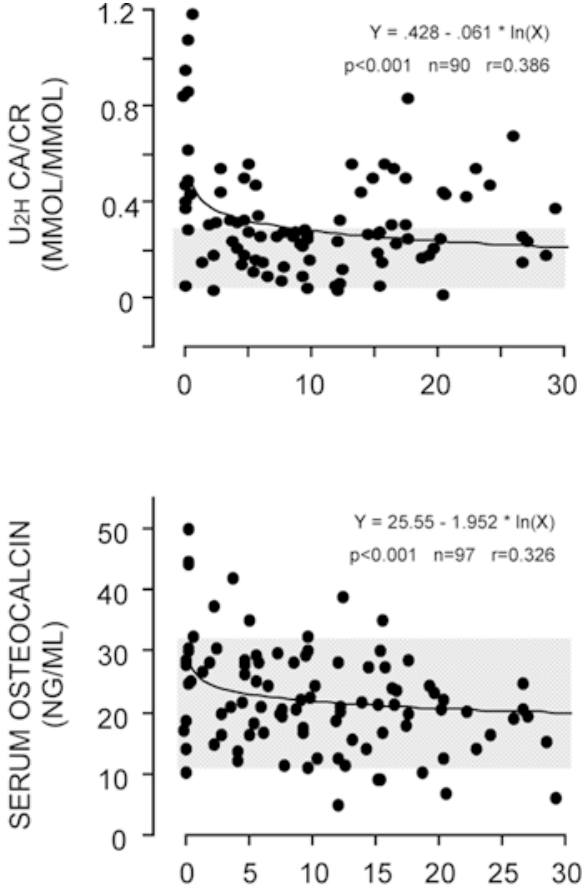

YEARS SINCE SPINAL CORD INJURY continuous demineralization which proceeded even beyond 10 years postinjury. This effect is previously undescribed, although it was recently suggested that bone loss at pelvis and legs could continue to decline linearly throughout the chronic phase of immobilization in male monozygotic twin pairs - one twin of each pair having SCI [13]. Other cross-sectional studies were either too small or too heterogeneous to detect the effect [11, $12,14]$.

The findings from quantitative ultrasound measurements were entirely consistent with BMD results, not only with respect to distinction of bone loss at cortical vs trabecular sublesional sites but also with respect to preservation of bone mass at the upper limbs. There was a high correlation between BMD at tibial epiphysis and QUI.CALC, and BMD at tibial diaphysis and SOS.TIB. Quantitative ultrasound is partly determined by bone mass. However, SOS is also a function of elasticity whereas BUA may be related to bone structure [18]. Whether the observed changes add the dimension of deterioration of bone quality to the BMD results in patients after SCI cannot be concluded from this study. However, these results are consistent with those from Chow et al. using an Achilles device at the calcaneus [16]. Studies using other than calcaneal devices are lacking.

The results on biochemical parameters suggest that an imbalance between elevated bone resorption and normal (to low) bone formation after SCI plays a role in the pathogenesis of bone loss and fracture in paraplegic men. Bone resorption was dramatically increased early ( $<1$ year) after SCI with an almost fivefold elevated mean urinary $\mathrm{D}-\mathrm{pyr} / \mathrm{Cr}$ and $\mathrm{Ca} / \mathrm{Cr}$, while bone formation lay within the (upper) reference range. Furthermore, in the acute stage (stratum I), there was no correlation between resorption and formation parameters, pointing to an uncoupling of the two processes. The fact that-despite counterregulatory suppression of serum intact $\mathrm{PTH}$, and increase of renal calcium excretion-frank hypercalcemia occurred in $50 \%$ of recently injured men (stratum I), indicates how excessively bone was resorbed. The present data are in accordance with the findings of a recent small longitudinal follow-up study over 6 months in para- and tetraplegics early after SCI [4]. Two older studies reported that hydroxyprolinuria returned towards baseline 6 to 12 months after SCI [24, 25]; another study suggested that high bone resorption was ongoing for up to 3 to 5 years after SCI, based on indirect evidence (suppressed PTH levels) in a cross-sectional setting [26]. As an unexpected finding, $30 \%$ of the paraplegics in strata III and IV (i.e., $>10$ years after SCI) showed an elevated bone resorption activity, and-similar to the finding in stratum I- the bone formation markers did not show a significant correlation with resorption markers at that chronic stage. Paraplegics of strata III + IV with high resorption markers had furthermore lower BMD $Z$-scores at the tibial sites and had approximately 4 times more often a history of fragility fracture compared with chronic paraplegics with normal D-pyr/Cr ratios. Thus, it can be assumed that in $30 \%$ of chronic paraplegics, excessive bone resorption had been persisting over a longer period of time and did not represent an accidental or transitory finding. Furthermore, paraplegics with a history of 
fracture had significantly lower osteocalcin serum levels compared with the nonfractured.

The BMD and QUS results of the present study show that bone loss after SCI is not a systemic process but a process restricted to unloaded skeletal sites. These clinical findings are in line with the hypothesis of Frost and others $[27,28]$ stating that mechanical unloading induces the increased bone resorption by suppressing an inhibitory control on osteoclasts and that - in the absence of mechanical strain - formation cannot cope with this increased bone resorption. Using human iliac bone marrow cultures of paraplegic patients, Demulder et al. [29] have recently shown an increase of osteoclast-like cell formation below the lesional level which they explained by locally elevated IL- 6 production arising from modifications in the bone marrow microenvironment in the paralyzed territories. Neurovascular changes secondary to the modification of the autonomic nervous system [24] with decreased local release of neuropeptides (such as calcitonin gene-related peptide) that inhibit bone resorption could directly contribute to the increased resorption activity after SCI [30].

This cross-sectional study documents the time course and the clinical consequences of bone loss after spinal cord injury in a homogeneous group of male paraplegics. Based on DXA, ultrasound, and biochemical measurements it seems reasonable to assume that infralesional bone loss is based on increased bone resorption with uncoupled bone formation and is a continuous process, at least in the cortical bone compartment. Based on the high burden of illness represented by fragility fractures in these patients, longitudinal studies should be designed to gain a better understanding of the pathophysiology of the disease and of possible treatment interventions.

Acknowledgements We thank Dr Philippe Kress for his invaluable contribution to the preparation of the manuscript.

\section{References}

1. Nottage WM (1981) A review of long-bone fractures in patients with spinal cord injury. Clin Orthop 155:65-70

2. Vestergaard P, Krogh K, Rejnmark L, Mosekilde L (1998) Fracture rates and risk factors for fractures in patients with spinal cord injury. Spinal Cord 36:790-796

3. Albright F, Burnett CH, Cope O, Parson W (1941) Acute atrophy of bone (osteoporosis) simulating hyperparathyroidism. J Clin Endocrinol Metab 1:711-716

4. Roberts D, Lee W, Cuneo RC et al (1998) Longitudinal study of bone turnover after acute spinal cord injury. J Clin Endocrinol Metab 83:415-422

5. Biering-Sorensen F, Bohr HH, Schaadt OP (1990) Longitudinal study of bone mineral content in the lumbar spine, the forearm and the lower extremities after spinal cord injury. Eur J Clin Invest 20:330-335

6. Garland DE, Stewart CA, Adkins RH et al (1992) Osteoporosis after spinal cord injury. J Orthop Res 10:371-378

7. Wilmet E, Ismail AA, Heilporn A, Welraeds D, Bergmann P (1995) Longitudinal study of the bone mineral content and of soft tissue composition after spinal cord section. Paraplegia 33:674-677
8. Frey-Rindova P, de Bruin ED, Stüssi E, Dambacher MA, Dietz V (2000) Bone mineral density in upper and lower extremities during 12 months after spinal cord injury measured by peripheral quantitative computed tomography. Spinal Cord 38:26-32

9. Minaire P, Meunier P, Edouard C, Bernard J, Courpron P, Bourret J (1974) Quantitative histological data on disuse osteoporosis. Calcif Tiss Res 17:57-73

10. Biering-Sorensen F, Bohr H, Schaadt O (1988) Bone mineral content of the lumbar spine and lower extremities years after spinal cord lesion. Paraplegia 26:293-301

11. Szollar SM, Martin EME, Sartoris DJ, Parthemore JG, Deftos LJ (1998) Bone mineral density and indexes of bone metabolism in spinal cord injury. Am J Phys Med Rehabil 77:28-35

12. Goemaere S, Van Laere M, De Neve P, Kaufman JM (1994) Bone mineral status in paraplegic patients who do or do not perform standing. Osteoporos Int 4:138-143

13. Bauman WA, Spungen AM, Wang J, Pierson RN, Schwartz E (1999) Continuous loss of bone during chronic immobilization: a monozygotic twin study. Osteoporos Int 10:123-127

14. Dauty M, Verbe BP, Maugars Y, Dubois C, Mathe JF (2000) Supralesional and sublesional bone mineral density in spinal cord injured patients. Bone 27:305-309

15. Ragnarsson KT, Sell HG (1981) Lower extremity fractures after spinal cord injury: a retrospective study. Arch Phys Med Rehabil 62:418-423

16. Chow YW, Inman C, Pollintine P et al (1996) Ultrasound bone densitometry and dual energy $\mathrm{x}$-ray absorptiometry in patients with spinal cord injury: a cross-sectional study. Spinal Cord 34:736-741

17. Casez JP, Troendle A, Lippuner K, Jaeger P (1994) Bone mineral density at distal tibia using dual-energy $\mathrm{x}$-ray absorptiometry in normal women and in patients with vertebral osteoporosis or primary hyperparathyroidism. J Bone Miner Res 9:1851-1857

18. Kaufman JJ, Einhorn TA (1993) Perspectives: ultrasound assessment of bone. J Bone Miner Res 8:517-525

19. Foldes A, Rimon A, Keinan D, Popovtzer M (1995) Quantitative ultrasound of the tibia: a novel approach for assessment of bone status. Bone 17:363-367

20. Joly J, Westhovens R, Borghs H et al (1999) Reference curve and diagnostic sensitivity for a new ultrasound device for the phalanges, the DBMsonic 1200, in Belgian women. Osteoporos Int 9:284-289

21. Eichenholtz SN (1963) Management of long-bone fractures in paraplegic patients. J Bone Joint Surg 45:299-310

22. Ingram RR, Suman RK, Freeman PA (1989) Lower limb fractures in the chronic spinal cord injured patient. Paraplegia 27:133-139

23. Comarr AE, Hutchinson RH, Borse E (1962) Extremity fractures of patients with spinal cord injuries. Am J Surg 103:732739

24. Bergmann P, Heilporn A, Schoutens A, Paternot J, Tricot A (1977) Longitudinal study of calcium and bone metabolism in paraplegic patients. Paraplegia 15:147-159

25. Chantraine A (1971) Clinical investigations of bone metabolism in spinal cord lesions. Paraplegia 8:253-259

26. Vaziri ND, Pandian MR, Segal JL, Winer RL, Eltorai I, Brunnemann S (1994) Vitamin D, parathormone, and calcitonine profiles in persons with long-standing spinal cord injury. Arch Phys Med Rehabil 75:766-769

27. Frost HM (1987) The mechanostat: a proposed pathogenic mechanism of osteoporosis and the bone mass effect of mechanical and non-mechanical agents. Bone Miner 2:73-85

28. Burger EH, Klein-Nuhlend J, Van Stien ME, Veldhuizen JP (1987) Inhibiting effect of mechanical stimulation on bone resorption in vivo. In: Christiansen C, Rijs B (eds) Osteoporosis. Osteopress, Copenhagen, pp 767770 
29. Demulder A, Guns M, Ismail A, Wilmet E, Fondu P, Bergmann P (1998) Increased osteoclast-like cells formation in longterm bone marrow cultures from patients with a spinal cord injury. Calcif Tissue Int 63:396-400
30. D’Souza SM, Mac Intyre I, Girgis SI, Mundy GR (1986) Human synthetic calcitonin gene-related peptide inhibits bone resorption in vitro. Endocrinology 119:58-61 